\title{
Action hamiltonienne d'un tore et formule de localisation en cohomologie équivariante
}

\author{
Paul-Emile Paradan
}

Résumé - Exploitant une idée de Witten [8], nous établissons une formule de localisation en cohomologie équivariante dans le cadre d'une action hamiltonienne d'un tore sur une variété symplectique compacte $\mathcal{X}$. L'intégrale sur $\mathcal{X}$ d'une forme équivariante fermée s'exprime comme une intégrale sur les points critiques de la fonction égale au carré de l'application moment.

\section{Hamiltonian torus action and localization formula in equivariant cohomology}

\begin{abstract}
Using an idea of Witten [8], we give a localization formula in equivariant cohomology in the case of an Hamiltonian torus action on a compact symplectic manifold $\mathcal{X}$. The integral over $\mathcal{X}$ of an equivariant closed form can be written as an integral over the submanifold of critical points of the square of the moment map.
\end{abstract}

Abridged English Version - Let $\mathcal{X}$ be a compact symplectic manifold equipped with an Hamiltonian action of a compact Lie group $G$, with Lie algebra $\mathfrak{g}$. We denote by $\Omega$ the symplectic form on $\mathcal{X}$ and $\mu: \mathcal{X} \rightarrow \mathfrak{g}^{*}$ the moment map of this action.

Let $\mathcal{H}_{G}^{\infty}(\mathcal{X})$ the $G$-equivariant cohomology of $\mathcal{X}$ with $\mathbb{C}^{\infty}$ coefficients (cf 1.1). Integration over $\mathcal{X}$, denoted $\int_{\mathcal{X}}$, induces a morphism $\int_{\mathcal{X}}: \mathcal{H}_{G}^{\infty}(\mathcal{X}) \rightarrow$ $\mathbb{C}^{\infty}(\mathfrak{g})^{G}$, where $\mathbb{C}^{\infty}(\mathfrak{g})^{G}$ denotes the algebra of smooth, $G$-invariant functions on $\mathfrak{g}$.

Let $\|$.$\| be a G$-invariant scalar product on $\mathfrak{g}$. In [8], Witten introduced the "partition functions" $Z(u)=\int_{\mathcal{X} \times \mathfrak{g}} \alpha(X) e^{i \Omega_{\mathfrak{g}}(X)} e^{-u\|X\|^{2} / 2} d X$ with $u>0$, where $\alpha(X)$ is a closed differential form which is polynomial in the variable $X$, and $\Omega_{\mathfrak{g}}(X)=\Omega+\langle\mu, X\rangle$ is the equivariant symplectic form.

Under the hypothesis that $G$ acts freely on $\mu^{-1}(0)$, Witten proposed a formula of the form $Z(u)=\sum_{N} Z_{N}(u)$, where " $N$ " ranges over the connected components of the subset $\operatorname{Cr}\left(\|\mu\|^{2}\right)$ of critical points of the square of the moment map. In this formula, each function $Z_{N}(u)$ should only depend of the value of the form $\alpha$ on $N$. Witten determined the function $Z_{\mu^{-1}(0)}$, which is in fact a polynomial, and gives for the other terms a bound of the form $Z_{N}(u)=O\left(e^{-k_{N} / u}\right), u>0$ with $k_{N}>0$. Witten's formula has been proved by Jeffrey and Kirwan [2], using different methods than Witten . 
One of the problems which makes the calculus of the functions $Z_{N}$ difficult is that, in the context of a general compact group, the subset $\operatorname{Cr}\left(\|\mu\|^{2}\right)$ is not a smooth submanifold of $\mathcal{X}$.

In the case of an Hamiltonian torus action, we work out the ideas of Witten to obtain a new kind of localization formula in equivariant cohomology. In this Note, we explain the ingredients of this formula.

Suppose now that the group $G=T$ is a torus with Lie algebra $t$. Consider the applications $\mu_{\varepsilon}=\mu-\varepsilon, \varepsilon \in \mathfrak{t}^{*}$. For $\varepsilon \in \mathfrak{t}^{*}$ generic, $\operatorname{Cr}\left(\left\|\mu_{\varepsilon}\right\|^{2}\right)$ is smooth and we have a decomposition $\operatorname{Cr}\left(\left\|\mu_{\varepsilon}\right\|^{2}\right)=\cup_{\Delta \in \mathcal{B}} C_{\Delta}^{\varepsilon}$, where $\mathcal{B}$ is a collection of affine subspaces of $\mathfrak{t}^{*}$ (cf. Proposition 2). Fix a generic $\varepsilon \in \mathfrak{t}^{*}$. Using the same process of localization as Witten, we show that for every $\eta \in \mathcal{H}_{T}^{\infty}(\mathcal{X})$ we have the equality

$$
\int_{\mathcal{X}} \eta=\sum_{\Delta \in \mathcal{B}} I_{\Delta}^{\varepsilon}(\eta)
$$

in the space $\mathcal{C}^{-\infty}(\mathfrak{t})$ of generalized functions on $\mathfrak{t}$ (cf. 1.1), where the generalized functions $I_{\Delta}^{\varepsilon}(\eta)$ have support contained in the subspace of $\mathfrak{t}$ orthogonal (for the duality) to the direction of the affine subspace $\Delta$, and depend only of the value of the form $\eta$ on the submanifold $C_{\Delta}^{\varepsilon}$ (cf. Théorème).

This localization permits us to give an explicit description of the asymptotic behaviour of the partition functions " $Z(u)$ " (cf. Proposition 3).

The reader will find the proofs of the propositions and the theorem in [6].

1. Cohomologie Équivariante À COEFFICIENTS GÉNÉRAlisés. - 1.1. Soit $\mathcal{X}$ une variété différentielle. On note $\mathcal{A}(\mathcal{X})$ l'algèbre sur $\mathbb{C}$ des formes différentielles et $d$ la dérivation extérieure. Si $\xi$ est un champ de vecteurs sur $\mathcal{X}$, on note $c(\xi): \mathcal{A}(\mathcal{X}) \rightarrow \mathcal{A}(\mathcal{X})$ la contraction par le champ de vecteurs $\xi$.

Soit $G$ un groupe de Lie, d'algèbre de Lie $\mathfrak{g}$, opérant sur $\mathcal{X}$. Cette action détermine un morphisme $X \rightarrow X_{\mathcal{X}}$ de $\mathfrak{g}$ dans l'algèbre des champs de vecteurs de $\mathcal{X}$.

Rappelons le modèle de De Rham pour la cohomologie $G$-équivariante de $\mathcal{X}[1,5]$. Soit $\mathbb{C}^{\infty}(\mathfrak{g}, \mathcal{A}(\mathcal{X}))$ l'algèbre des formes $\alpha(X)$ sur $\mathcal{X}$ dépendant de manière $\mathbb{C}^{\infty}$ de $X \in \mathfrak{g}$. Nous noterons $\mathcal{A}_{G}^{\infty}(\mathcal{X})$ la sous-algèbre de $\mathbb{C}^{\infty}(\mathfrak{g}, \mathcal{A}(\mathcal{X}))$ formée des éléments $G$-invariants. On définit l'opérateur $\mathcal{D}$ sur $\mathcal{A}_{G}^{\infty}(\mathcal{X})$ par $(\mathcal{D} \alpha)(X):=\left(d-c\left(X_{\mathcal{X}}\right)\right)(\alpha(X))$. On remarque que $\mathcal{D}^{2}=0$ sur $\mathcal{A}_{G}^{\infty}(\mathcal{X})$. La cohomologie du complexe $\left(\mathcal{A}_{G}^{\infty}(\mathcal{X}), \mathcal{D}\right)$ est appelée la cohomologie $G$ équivariante (à coefficients $\mathbb{C}^{\infty}$ ) de $\mathcal{X}$, et notée $\mathcal{H}_{G}^{\infty}(\mathcal{X})$.

Soit $\mathcal{C}^{-\infty}(\mathfrak{g}, \mathcal{A}(\mathcal{X}))$ l'espace des fonctions généralisées sur $\mathfrak{g}$ à valeurs formes différentielles sur $\mathcal{X}$. On note $\mathcal{A}_{G}^{-\infty}(\mathcal{X})$ le sous-espace des éléments 
$G$-invariants de $\mathcal{C}^{-\infty}(\mathfrak{g}, \mathcal{A}(\mathcal{X}))$. On remarque que $\mathcal{A}_{G}^{\infty}(\mathcal{X}) \subset \mathcal{A}_{G}^{-\infty}(\mathcal{X})$ et on note encore $\mathcal{D}$ la différentielle sur $\mathcal{A}_{G}^{-\infty}(\mathcal{X})$ qui prolonge celle de $\mathcal{A}_{G}^{\infty}(\mathcal{X})$ (pour la définition voir [5]). La cohomologie du complexe $\left(\mathcal{A}_{G}^{-\infty}(\mathcal{X}), \mathcal{D}\right)$ est appelée la cohomologie $G$-équivariante (à coefficients $\mathcal{C}^{-\infty}$ ) de $\mathcal{X}$, et est notée $\mathcal{H}_{G}^{-\infty}(\mathcal{X})$.

On note $\mathcal{C}^{-\infty}(\mathfrak{g})^{G}$ l'espace des fonctions généralisées sur $\mathfrak{g}$ invariantes par $G$. Si $\mathcal{X}$ est une $G$-variété compacte orientée, l'intégration $\alpha \rightarrow \int_{\mathcal{X}} \alpha(X)$ sur $\mathcal{X}$ est une application $\int_{\mathcal{X}}$ de $\mathcal{A}_{G}^{-\infty}(\mathcal{X})$ dans $\mathcal{C}^{-\infty}(\mathfrak{g})^{G}$. Cette application envoie le sous-espace $\mathcal{A}_{G}^{\infty}(\mathcal{X})$ dans l'algèbre $\mathbb{C}^{\infty}(\mathfrak{g})^{G}$ des fonctions $\mathbb{C}^{\infty}$ sur $\mathfrak{g}$ qui sont $G$-invariantes.

1.2. Soit $\mathcal{E} \rightarrow M$ un $G$-fibré euclidien orienté sur une variété $M$, muni d'une connexion linéaire euclidienne $G$-invariante $\nabla^{\mathcal{E}}$. On note $\mathcal{A}(M, \mathcal{E})=$ $\Gamma\left(M, \wedge T^{*} M \otimes \mathcal{E}\right)$ l'espace des formes différentielles sur $M$ à valeurs dans $\mathcal{E}$. Au moyen de la connexion $\nabla^{\mathcal{E}}$, on définit le moment de $X \in \mathfrak{g}$ : c'est un opérateur $\mu^{\mathcal{E}}(X) \in \mathcal{A}(M, \operatorname{so}(\mathcal{E})) \subset \operatorname{End}(\mathcal{A}(M, \mathcal{E})$ ) (Définition 7.5., [1]). Ici $\operatorname{so}(\mathcal{E})$ désigne le sous-fibré de $\mathcal{E} \otimes \mathcal{E}^{*}$ formé des endomorphismes antisymétriques de $\mathcal{E}$. Définissons maintenant quelques formes équivariantes.

Si $R^{\mathcal{E}} \in \mathcal{A}(M, \operatorname{so}(\mathcal{E}))$ est la courbure de la connexion $\nabla^{\mathcal{E}}$, la courbure équivariante de $\nabla^{\mathcal{E}}$ est l'application de $\mathfrak{g}$ dans $\mathcal{A}(M, \operatorname{so}(\mathcal{E}))$ définie par $X \rightarrow$ $R_{\mathfrak{g}}^{\mathcal{E}}(X)=\mu^{\mathcal{E}}(X)+R^{\mathcal{E}}$.

Considérons le pfaffien $\operatorname{det}_{o}^{1 / 2}: \mathcal{A}(M, \mathrm{so}(\mathcal{E})) \rightarrow \mathcal{A}(M)$. On définit la forme d'Euler équivariante de $\left(\mathcal{E}, \nabla^{\mathcal{E}}\right)$ en posant $\operatorname{Eul}_{o}\left(\mathcal{E}, \nabla^{\mathcal{E}}\right)(X)$ $=\operatorname{det}_{o}^{1 / 2}\left(\frac{-1}{2 \pi} R_{\mathfrak{g}}^{\mathcal{E}}(X)\right)$. On démontre que $\operatorname{Eul}_{o}\left(\mathcal{E}, \nabla^{\mathcal{E}}\right)$ est une forme équivariante fermée à coefficients polynomiaux, et que sa classe de cohomologie ne dépend ni de la connexion, ni du produit scalaire sur les fibres (voir [1], chapitre 7).

Supposons que $G=T$ est un tore et notons $\mathfrak{t}$ son algèbre de Lie. On suppose que les points fixes de l'action de $T$ sur $\mathcal{E}$ sont les points de la variété $M: \mathcal{E}^{T}=M$. Dans ce cadre, nous allons inverser la forme d'Euler équivariante dans $\mathcal{A}_{T}^{-\infty}(M)$. Soit $\beta \in \mathfrak{t}$ tel que le champ de vecteurs engendré $\operatorname{par} \beta$ sur $\mathcal{E}$ s'annule exactement sur $M$. On constate que pour tout $s>0$ et tout $X \in \mathfrak{t}$ la forme $\operatorname{Eul}_{o}\left(\mathcal{E}, \nabla^{\mathcal{E}}\right)(X+i s \beta)$ possède une composante de degré 0 qui ne s'annule pas sur $M$; on peut donc l'inverser dans $\mathcal{A}(M)$. On vérifie que pour tout $s>0, X \rightarrow A_{s}(X)=\operatorname{Eul}_{o}\left(\mathcal{E}, \nabla^{\mathcal{E}}\right)(X+i s \beta)^{-1}$ est une forme équivariante fermée sur $M$. En passant à la limite sur $s$ nous obtenons la

Proposition 1 La famille $\left(A_{s}\right)_{s>0}$ de formes équivariantes (à coefficients $\left.\mathbb{C}^{\infty}\right)$ sur $M$ converge, lorsque $s \rightarrow 0$, vers une forme équivariante fermée $\operatorname{Eul}_{\beta}^{-1}\left(\mathcal{E}, \nabla^{\mathcal{E}}\right) \in \mathcal{A}_{T}^{-\infty}(M)$.

1.3. Soit $P$ une variété compacte sur laquelle un tore $T$ agit localement librement. On considère le quotient $M:=P / T$ qui possède une structure 
de $\mathrm{V}$-variété [4]. Les formes différentielles de $M$ sont définies comme les éléments $T$-basiques de l'algèbre $\mathcal{A}(P)$. Ces éléments forment une sousalgèbre $\mathcal{A}(M)$ qui est stable par rapport à la différentiation extérieure: on note $\mathcal{H}(M)$ la cohomologie du complexe $(\mathcal{A}(M), d)$. Le tore agit trivialement sur $M$, ainsi $\mathcal{A}_{T}^{-\infty}(M):=\mathcal{C}^{-\infty}(\mathfrak{t}, \mathcal{A}(M))$ et la cohomologie associée, $\mathcal{H}_{T}^{-\infty}(M)$, est égale à $\mathcal{C}^{-\infty}(\mathfrak{t}, \mathcal{H}(M))$.

Soit $\omega \in \mathcal{H}^{2}(M) \otimes \mathfrak{t}$ la courbure du V-fibré principal $P \rightarrow M$. On note $\delta(X-\omega) \in \mathcal{H}_{T}^{-\infty}(M)$ la classe de support 0 définie par $<\delta(X-$ $\omega), \phi(X) d X>:=\phi(\omega) \operatorname{vol}(T, d X)$, pour toute fonction $\phi \in \mathbb{C}^{\infty}(\mathfrak{t})$. Dans cette formule $d X$ est une mesure euclidienne pour $\mathfrak{t}$ et $\operatorname{vol}(T, d X)$ est le volume du groupe $T$ pour la mesure de Haar compatible avec $d X$.

2. Action hamiltonienne et points CRitiques De $\|\mu\|^{2}$. - Soit $(\mathcal{X}, \Omega)$ une variété symplectique compacte munie de l'action hamiltonienne d'un tore $T$. On note $\mathfrak{t}$ l'algèbre de Lie de $T$ et $\mathfrak{t}^{*}$ l'espace vectoriel dual. On note $\mu: \mathcal{X} \rightarrow \mathfrak{t}^{*}$ l'application moment; c'est une application $T$-équivariante qui vérifie $c\left(X_{\mathcal{X}}\right) \Omega=d\langle\mu, X\rangle, X \in \mathfrak{t}$. Pour tout $\varepsilon \in \mathfrak{t}^{*}$, on note $\mu_{\varepsilon}$ l'application $\mu-\varepsilon$ (c'est encore une application moment).

Comme la variété $\mathcal{X}$ est compacte, l'action de $T$ sur $\mathcal{X}$ possède un nombre fini de types d'orbites: soient $T_{1}, \ldots, T_{r}$ les sous-groupes de $T$, stabilisateurs de points de $\mathcal{X}$. Pour chaque $l=1, \ldots, r$ on note $\mathcal{Z}_{l}^{k}, k=1, \ldots, n_{l}$ les composantes connexes de $\mathcal{X}^{T_{l}}$ qui ont pour stabilisateur générique le sousgroupe $T_{l}$. Les $\mathcal{Z}_{l}^{k}$ sont des sous-variétés symplectiques $T$-invariantes de $\mathcal{X}$. Le théorème de convexité d'Atiyah-Guillemin-Sternberg assure que les $P_{l k}:=\mu\left(\mathcal{Z}_{l}^{k}\right)$ sont des polytopes de $\mathfrak{t}^{*}$. On a $\left(\overrightarrow{P_{l k}}\right)^{\perp}=\operatorname{Lie}\left(T_{l}\right), \quad \forall k=$ $1, \ldots, n_{l}$. Dans cette égalité $\overrightarrow{P_{l k}}$ est le sous-espace vectoriel engendré par $\left\{a-b \mid a, b \in P_{l k}\right\}$ et ${ }^{\perp}$ désigne l'orthogonal pour la dualité entre $\mathfrak{t}$ et $\mathfrak{t}^{*}$.

On note $\mathcal{B}:=\left\{\operatorname{Aff}\left(P_{l k}\right) \mid l, k\right\}$ l'ensemble des sous-espaces affines de $\mathfrak{t}^{*}$ engendrés par les polytopes $P_{l k}$. Pour chaque $\Delta \in \mathcal{B}$, on note $T_{\Delta}$ le sous-tore de $T$ d'algèbre de Lie $(\vec{\Delta})^{\perp}$.

On munit $\mathfrak{t}^{*}$ d'un produit scalaire et on note $j: \mathfrak{t} \rightarrow \mathfrak{t}^{*}$ l'isomorphisme induit par ce produit scalaire. Pour tout $\varepsilon \in \mathfrak{t}^{*}$, nous considérons la fonction $\left\|\mu_{\varepsilon}\right\|^{2}$ de $\mathcal{X}$ dans $\mathbb{R}$. En nous inspirant du premier chapitre de [3], nous obtenons la

Proposition 2 Pour tout $\varepsilon \in \mathfrak{t}^{*}$, les points critiques de $\left\|\mu_{\varepsilon}\right\|^{2}$ se mettent sous la forme

$$
\operatorname{Cr}\left(\left\|\mu_{\varepsilon}\right\|^{2}\right)=\bigcup_{\Delta \in \mathcal{B}} \mathcal{X}^{T_{\Delta}} \cap \mu^{-1}(\beta(\varepsilon, \Delta)),
$$

où $\beta(\varepsilon, \Delta)$ est le projeté orthogonal de $\varepsilon$ sur $\Delta$. Il existe un ouvert dense 
$W$ de $\mathfrak{t}^{*}$ tel que pour tout $\varepsilon \in W$ l'ensemble $\operatorname{Cr}\left(\left\|\mu_{\varepsilon}\right\|^{2}\right)$ est une sous-variété de $\mathcal{X}$, l'union précédente est disjointe, et le groupe $T / T_{\Delta}$ agit localement librement sur $C_{\Delta}^{\varepsilon}:=\mathcal{X}^{T_{\Delta}} \cap \mu^{-1}(\beta(\varepsilon, \Delta))$.

3. Localisation sur les points CRitiques de $\left\|\mu_{\varepsilon}\right\|^{2}$.- 3.1. On fixe $\varepsilon \in W$. Pour chaque $\Delta \in \mathcal{B}$, nous faisons le choix d'un supplémentaire de $\mathfrak{t}_{\Delta}$ dans $\mathfrak{t}$ que l'on note $\mathfrak{t} / \mathfrak{t}_{\Delta}$, tel que l'on ait une décomposition $T=T_{\Delta} \times T / T_{\Delta}$ où $T / T_{\Delta}$ désigne le sous-tore de $T$ d'algèbre de Lie $\mathfrak{t} / \mathfrak{t}_{\Delta}$.

Comme le groupe $T / T_{\Delta}$ agit localement librement sur la variété $C_{\Delta}^{\epsilon}$, on peut définir la $\mathrm{V}$-variété quotient $\mathcal{M}_{\Delta}^{\varepsilon}:=C_{\Delta}^{\varepsilon} / T / T_{\Delta}$ [4]. Nous avons le morphisme de Kirwan $k_{\Delta}: \mathcal{H}_{T}^{\infty}(\mathcal{X}) \rightarrow \mathcal{H}_{T_{\Delta}}^{\infty}\left(\mathcal{M}_{\Delta}^{\varepsilon}\right)$, défini comme le composé du morphisme de restriction $\mathcal{H}_{T}^{\infty}(\mathcal{X}) \rightarrow \mathcal{H}_{T}^{\infty}\left(C_{\Delta}^{\varepsilon}\right)$ et de l'isomorphisme de Chern-Weil $\mathcal{H}_{T}^{\infty}\left(C_{\Delta}^{\varepsilon}\right) \stackrel{\sim}{\rightarrow} \mathcal{H}_{T_{\Delta}}^{\infty}\left(\mathcal{M}_{\Delta}^{\varepsilon}\right)$.

A chaque composante connexe $F$ de $C_{\Delta}^{\varepsilon}$ on associe le groupe $S^{\Delta}(F)$ qui est le stabilisateur générique de $T / T_{\Delta}$ sur $F$ et on note $\left|S^{\Delta}\right|(F)$ son cardinal. L'application $F \rightarrow\left|S^{\Delta}\right|(F)$ détermine une fonction localement constante sur $\mathcal{M}_{\Delta}^{\varepsilon}$ qui sera notée $\left|S^{\Delta}\right|$.

Soient $\beta_{\Delta}:=j^{-1}(\beta(\varepsilon, \Delta)-\varepsilon) \in \mathfrak{t}_{\Delta}$ et $N_{\Delta}$ le fibré normal de $\mathcal{X}^{T_{\Delta}}$ dans $\mathcal{X}$ restreint à $C_{\Delta}^{\epsilon}$. Lorsque $\varepsilon \in W$, le champ de vecteurs sur $N_{\Delta}$ engendré par $\beta_{\Delta}$ s'annule exactement sur $C_{\Delta}^{\epsilon}$. Ces données nous permettent de définir, au moyen d'une connexion $\nabla^{N_{\Delta}}$, une forme $T_{\Delta}$-équivariante fermée à coefficients généralisés sur $C_{\Delta}^{\varepsilon}: \operatorname{Eul}_{\beta_{\Delta}}^{-1}\left(N_{\Delta}, \nabla^{N_{\Delta}}\right) \in \mathcal{A}_{T_{\Delta}}^{-\infty}\left(C_{\Delta}^{\varepsilon}\right)(\mathrm{cf}$. Proposition 1). En choisissant la connexion $T / T_{\Delta}$-horizontale on voit que $\operatorname{Eul}_{\beta_{\Delta}}^{-1}\left(N_{\Delta}, \nabla^{N_{\Delta}}\right) \in \mathcal{C}^{-\infty}\left(\mathfrak{t}_{\Delta}, \mathcal{A}\left(\mathcal{M}_{\Delta}^{\varepsilon}\right)\right):=\mathcal{A}_{T_{\Delta}}^{-\infty}\left(\mathcal{M}_{\Delta}^{\varepsilon}\right)$. On notera $\operatorname{Eul}_{\beta_{\Delta}}^{-1}\left(\mathcal{E}_{\Delta}\right) \in$ $\mathcal{H}_{T_{\Delta}}^{-\infty}\left(\mathcal{M}_{\Delta}^{\varepsilon}\right)$ la classe de cette forme équivariante. La notation $\mathcal{E}_{\Delta}$ fait référence au V-fibré $T_{\Delta}$-équivariant $\mathcal{E}_{\Delta}:=N_{\Delta} / T / T_{\Delta} \rightarrow \mathcal{M}_{\Delta}^{\varepsilon}$.

Le V-fibré principal $C_{\Delta}^{\varepsilon} \rightarrow \mathcal{M}_{\Delta}^{\varepsilon}$ de courbure $\omega_{\Delta}$ fournit, d'aprés 1.3., la classe $\delta\left(X-\omega_{\Delta}\right) \in \mathcal{H}_{T / T_{\Delta}}^{-\infty}\left(\mathcal{M}_{\Delta}^{\varepsilon}\right)$. La décomposition $\mathfrak{t}=\mathfrak{t}_{\Delta} \oplus \mathfrak{t} / \mathfrak{t}_{\Delta}$ induit une application bilinéaire $(\eta, \nu) \mapsto \eta \diamond \nu, \mathcal{H}_{T_{\Delta}}^{-\infty}\left(\mathcal{M}_{\Delta}^{\varepsilon}\right) \times \mathcal{H}_{T / T_{\Delta}}^{-\infty}\left(\mathcal{M}_{\Delta}^{\varepsilon}\right) \rightarrow$ $\mathcal{H}_{T}^{-\infty}\left(\mathcal{M}_{\Delta}^{\varepsilon}\right)$. La V-variété $\mathcal{M}_{\Delta}^{\varepsilon}$ possède une structure symplectique héritée de celle de $\mathcal{X}$. Elle est donc orientée et l'intégration sur $\mathcal{M}_{\Delta}^{\varepsilon}$, notée $\int_{\mathcal{M}_{\Delta}^{\varepsilon}}$, définit un morphisme de $\mathcal{H}_{T}^{-\infty}\left(\mathcal{M}_{\Delta}^{\varepsilon}\right)$ dans $\mathcal{C}^{-\infty}(\mathfrak{t})$.

On peut maintenant énoncer le résultat principal de cette Note:

Théorème Soient $\varepsilon \in W$ et $\eta \in \mathcal{A}_{T}^{\infty}(\mathcal{X})$ une forme fermée. Nous avons dans $\mathcal{C}^{-\infty}(\mathfrak{t})$ l'égalité

$$
\int_{\mathcal{X}} \eta=\sum_{\Delta \in \mathcal{B}} I_{\Delta}^{\varepsilon}(\eta)
$$


où $I_{\Delta}^{\varepsilon}(\eta)$ est la fonction généralisée de support $\mathfrak{t}_{\Delta}$ définie par

$I_{\Delta}^{\varepsilon}(\eta)\left(X_{1}+X_{2}\right)=(2 i \pi)^{\operatorname{dim} \Delta} \int_{\mathcal{M}_{\Delta}^{\varepsilon}} \frac{1}{\left|S^{\Delta}\right|} k_{\Delta}(\eta)\left(X_{1}\right) \operatorname{Eul}_{\beta_{\Delta}}^{-1}\left(\mathcal{E}_{\Delta}\right)\left(X_{1}\right) \diamond \delta\left(X_{2}-\omega_{\Delta}\right)$.

Dans cette formule les variables $X_{1}$ et $X_{2}$ sont dans $\mathfrak{t}_{\Delta}$ et $\mathfrak{t} / \mathfrak{t}_{\Delta}$.

Pour toute fonction $\phi \in \mathbb{C}^{\infty}(\mathfrak{t})$ à support compact, nous avons

$\int_{\mathcal{X} \times \mathfrak{t}} \eta(X) \phi(X) d X=\sum_{\Delta \in \mathcal{B}} c_{\Delta} \int_{\mathcal{M}_{\Delta}^{\varepsilon} \times \mathfrak{t}_{\Delta}} \frac{1}{\left|S^{\Delta}\right|} k_{\Delta}(\eta . \phi)\left(X_{1}\right) \operatorname{Eul}_{\beta_{\Delta}}^{-1}\left(\mathcal{E}_{\Delta}\right)\left(X_{1}\right) d X_{1}$.

avec $c_{\Delta}=(2 i \pi)^{\operatorname{dim} \Delta} \operatorname{vol}\left(T / T_{\Delta}, d X_{2}\right)$ et $d X=d X_{1} d X_{2}$.

Le calcul de la fonction généralisée $I_{\mathbf{t}^{*}}^{0}$ est effectué dans [2] et [7], sous l'hypothèse que le groupe $T$ agit localement librement sur $\mu^{-1}(0)$ (dans ce cas $\left.\mathfrak{t}^{*} \in \mathcal{B}\right)$.

Donnons quelques indications sur la démonstration de ce théorème. On fixe $\varepsilon \in W$. Soit $\eta$ une forme équivariante fermée de $\mathcal{X}$. Pour tout nombre complexe $z$ et toute forme équivariante $\lambda$ de degré impair l'exponentielle $e^{z \mathcal{D} \lambda}$ est définie sans ambiguité et nous avons $\int_{\mathcal{X}} \eta=\int_{\mathcal{X}} \eta e^{z \mathcal{D} \lambda}$.

Considérons, comme dans [8], le champ de vecteurs hamiltonien $\mathcal{H}^{\varepsilon}$ de la fonction $T$-invariante $\frac{1}{2}\left\|\mu_{\varepsilon}\right\|^{2}$. Au moyen d'une métrique riemannienne $T$-invariante $\operatorname{sur} \mathcal{X},(\cdot, \cdot)_{\mathcal{X}}$, on introduit la 1 -forme $\lambda^{\varepsilon}$ définie par $\lambda^{\varepsilon}:=$ $\left(\mathcal{H}^{\varepsilon}, \cdot\right) \mathcal{X}$. Le paramètre $z$ est choisi imaginaire pur: $z=-i s, s \in \mathbb{R}$. Le théorème de localisation provient de l'étude du comportement asymptotique de la forme $\eta e^{-i s \mathcal{D} \lambda^{\varepsilon}}$ lorsque $s \rightarrow+\infty$.

3.2. Voici une application de cette formule de localisation. Dans [8], Witten introduit les fonctions de partition " $Z$ " définies par

$$
Z(u):=\int_{\mathcal{X} \times \mathfrak{t}} \alpha(X) e^{i \Omega_{\mathfrak{t}}(X)} e^{-u\|X\|^{2} / 2} d X, \quad u>0,
$$

où $\alpha(X) \in \mathcal{A}_{T}^{\infty}(\mathfrak{t}, \mathcal{X})$ une forme équivariante fermée qui est polynomiale par rapport à $X \in \mathfrak{t}$, et $\Omega_{\mathfrak{t}}(X)=\Omega+\langle\mu, X\rangle$ est la forme symplectique équivariante.

En supposant que 0 est une valeur régulière de l'application moment, Witten donne les premiers termes du développement asymptotique de $Z(u)$ lorsque $u \rightarrow 0: Z(u)=Z_{\mu^{-1}(0)}(u)+O\left(e^{-\rho / u}\right)$ avec $\rho>0$ et où $Z_{\mu^{-1}(0)}(u)$ est un polynôme défini par

$$
u \in \mathbb{R}, \quad Z_{\mu^{-1}(0)}(u)=(2 i \pi)^{\operatorname{dim} T} \frac{\operatorname{vol}(T, d X)}{\left|S^{0}\right|} \int_{\mathcal{M}_{0}} k_{0}\left(\alpha e^{i \Omega_{\mathrm{t}}}\right) e^{-u\left\|\omega_{0}\right\|^{2} / 2} .
$$


Dans cette formule, $\mathcal{M}_{0}:=\mu^{-1}(0) / T$ est la réduction de Marsden-Weinstein en $0 ; \omega_{0}$ est la courbure du V-fibré principal $\mu^{-1}(0) \rightarrow \mathcal{M}_{0}$; et $k_{0}: \mathcal{H}_{T}^{\infty}(\mathcal{X}) \rightarrow$ $\mathcal{H}\left(\mathcal{M}_{0}\right)$ est l'application de Kirwan [3].

$\mathrm{Au}$ moyen du théorème précédent nous obtenons une description précise du comportement asymptotique des fonctions de partition.

Proposition 3 Supposons que 0 est une valeur régulière de $\mu$. On note $d_{\alpha}$ le degré polynomial de la forme $\alpha$. Il existe des fonctions $h_{\Delta}: \mathbb{R} \rightarrow \mathbb{C}$, $\mathbb{C}^{\infty}$ et paires, telles que pour tout $u>0$

$$
Z(u)=Z_{\mu^{-1}(0)}(u)+\sum_{\substack{\Delta \in \mathcal{B} \\ \Delta \neq \mathfrak{t}^{*}}} u^{n_{\Delta}} e^{-\frac{\left(\rho_{\Delta}\right)^{2}}{2 u}} h_{\Delta}(\sqrt{u}),
$$

où $n_{\Delta}=\frac{d i m T_{\Delta}}{2}-d_{\alpha}$ et $\rho_{\Delta}$ est la distance du point 0 au sous-espace affine $\Delta$.

Le lecteur intéressé pourra trouver les démonstrations complètes des propositions et du théorème dans [6].

\section{References}

[1] N. Berline, E. Getzler et M. Vergne, Heat kernels and Dirac operators, Grundlehren, vol. 298, Springer, Berlin, 1991.

[2] L. JefFrey et F. KIRWAN, Localization for non Abelian group action, Topology, 34, p. 291-327, 1995.

[3] F. Kirwan, Cohomology of quotients in symplectic and algebraic geometry, Princeton Univ. Press, Princeton, 1984.

[4] T. Kawasaki, The signature theorem for V-manifold, Topology, 17, 1978, p. 75-83.

[5] S. Kumar et M. Vergne, Equivariant cohomology with generalized coefficients, Astrisque, 215, 1993, p. 109-204.

[6] P.-E. Paradan, Formule de localisation en cohomologie équivariante, Thèse, Université Paris 7-Denis Diderot, 1996.

[7] M. Vergne, A note on the Jeffrey-Kirwan-Witten localization formula, Topology, 34, 1996, p. 243-266.

[8] E. Witten, Two dimensional gauge theories revisited, J. Geom. Phys. 9, 1992, p. 303-368. 